\title{
Magneto-optical control of light collapse in bulk Kerr media
}

\author{
Y. Linzon*, ${ }^{1}$ K. A. Rutkowska,,${ }^{1,2}$ B. A. Malomed, ${ }^{3}$ and R. Morandotti ${ }^{1}$ \\ ${ }^{1}$ Université du Quebec, Institute National de la Recherche Scientifique, Varennes, Quebec J3X 1S2, Canada \\ ${ }^{2}$ Faculty of Physics, Warsaw University of Technology, Warsaw PL-00662, Poland \\ ${ }^{3}$ Department of Physical Electronics, School of Electrical Engineering, \\ Faculty of Engineering, Tel Aviv University, Tel Aviv 69978, Israel
}

(Dated: November 3, 2018)

\begin{abstract}
Magneto-optical crystals allow an efficient control of the birefringence of light via the CottonMouton and Faraday effects. These effects enable a unique combination of adjustable linear and circular birefringence, which, in turn, can affect the propagation of light in nonlinear Kerr media. We show numerically that the combined birefringences can accelerate, delay, or arrest the nonlinear collapse of $(2+1) \mathrm{D}$ beams, and report an experimental observation of the acceleration of the onset of collapse in a bulk Yttrium Iron Garnet (YIG) crystal in an external magnetic field.
\end{abstract}

PACS numbers: 42.65.Sf, 42.65.Jx, 42.81.Gs, 78.20.Ls

Waves propagating in multidimensional self-focusing media are subject to instabilities that lead to the catastrophic collapse after a finite propagation distance, followed by beam filamentation or material damage [1, 2, 3, 4, 5, 6, 7, 8, 9, 10, 11]. In the $(2+1) \mathrm{D}[(2+1)-$ dimensional)] setting, above a certain threshold value of the input power, the critical collapse driven by focusing nonlinearities is a universal scenario, observed in high-power excitations of plasmas [1, 2], hydrodynamical systems [3], Bose-Einstein condensates (BECs) [4, [5], and optical media [2, 6, 7]. In particular, in optical pulse propagation through amorphous media and crystals without special symmetries, the dominant nonlinearity is the Kerr (cubic) effect, which is modeled by the nonlinear Schrödinger equation (NLSE) [12]. Collapsing beams in Kerr media were studied in detail, especially in the course of the past decade $[6,7,6,8,9,10,11]$.

The challenge to control the wave collapse (and in particular to mitigate its detrimental effects) has recently drawn much attention [5, 9, 10, 11]. As recently demonstrated, the collapse distance of ultra-intense laser pulses in air can be controlled by passive optical elements [9], and in BECs the collapse time is strongly affected by the so-called Feshbach-resonance technique [5]. In condensed optical media, where the collapse occurs with pulse energies far below the creation of plasma [6, 7], a recently proposed scheme for collapse management relies on the use of a layered structure with the nonlinearity strength alternating in the longitudinal direction [10] (a similar mechanism was proposed for the stabilization of BECs in the $2 \mathrm{D}$ case [1] ). However, this scheme is difficult to implement in bulk media, and such structures may give rise to linear losses induced by the reflection of light from interfaces between the alternating layers.

An alternative approach to control the transition to the collapse may be provided by optical birefringence, which promotes energy and phase transfer between the polarization components of the beam. The interplay between birefringence and nonlinearity is known to induce coupling of the polarization rotation and a characteristic temporal evolution of solitons in optical fibers [13]. In this paper, we explore the birefringence as a tool for the "management" of the collapse of $(2+1) \mathrm{D}$ beams, which may be relatively easily implemented in affordable experimental conditions by using magneto-optical (MO) effects 14, 15, 16, 17], while avoiding reflection losses. To this end, we present numerical and experimental studies of the combined effects of linear and circular birefringences on the dynamics of collapsing beams in a bulk self-focusing Kerr medium. We find that the onset of collapse can be accelerated, delayed, or even suppressed, at certain values of the combined birefringence strengths. We also show that the required linear and circular birefringence can be induced in a transparent MO Yttrium Iron Garnet (YIG, $\mathrm{Y}_{3} \mathrm{Al}_{5} \mathrm{O}_{12}$ ) crystal by the application of an external dc magnetic field, thus opening up the perspective of using MO effects to generate and control various nonlinear phenomena in optics. In our experiments, an adjustable balance of linear and circular birefringences was realized via a combination [14] of the Cotton-Mouton (or Voigt) [15] and Faraday [16] MO effects in a bulk YIG crystal. Following the propagation of femtosecond pulses in the crystal, we observed a controllable decrease of the threshold input power necessary for the onset of collapse at the output facet as a function of the magnetic field. This also constitutes a first experimental study in nonlinear optics where birefringence can be switched on and varied continuously in an adjustable fashion.

The evolution of the complex electric-field amplitudes, $u_{r}$ and $u_{l}$, representing the right- and left-circular polarizations (RCP and LCP), in the presence of a Kerr nonlinearity and combined linear and circular birefringences, obeys the coupled NLSEs, in the scaled form [13]:

$$
\begin{gathered}
i \frac{\partial u_{r}}{\partial z}+\frac{1}{2} \nabla_{\perp}^{2} u_{r}+b u_{r}+c u_{l}+\left(\left|u_{r}\right|^{2}+2\left|u_{l}\right|^{2}\right) u_{r}=0 \\
i \frac{\partial u_{l}}{\partial z}+\frac{1}{2} \nabla_{\perp}^{2} u_{l}-b u_{l}+c u_{r}+\left(\left|u_{l}\right|^{2}+2\left|u_{r}\right|^{2}\right) u_{l}=0
\end{gathered}
$$

where $z$ is the propagation axis, $\nabla_{\perp}^{2}$ is the transverse 
Laplacian, while $b$ and $c$ are the strengths of the circular and linear birefringences, respectively. As evident from Eqs. (1) the linear and circular birefringences account for, respectively, the rates of linear amplitude mixing and phase shift between the RCP and LCP fields. In terms of the RCP and LCP, the ratio between the cross- and self-phase modulation coefficients (XPM/SPM) is 2 for cubically-symmetric crystals, including YIG [12, 13].

Assuming vorticity-free solutions with circular symmetry, $(2+1) \mathrm{D}$ Eqs. (1) reduce to a $(1+1) \mathrm{D}$ form, in terms of $z$ and the radial coordinate $R$. We consider input Gaussian beams, $u_{r}(R, z=0)=A \exp \left(-\frac{R^{2}}{2 \rho^{2}}\right) \cos \theta$ and $u_{l}(R, z=0)=A \exp \left(-\frac{R^{2}}{2 \rho^{2}}\right) \sin \theta$, with normalized input width $\rho=1$, amplitude $A$, and symmetric polarization content $\theta=\pi / 4$. This choice corresponds to an unchirped Gaussian profile launched with an horizontal linear polarization. Since the input does not carry vorticity, the circular-symmetric structure of the solutions is not subject to an azimuthal modulational instability [7].

Figure 1 shows propagation maps obtained by direct simulations of Eqs. (11). In the absence of birefringence [Figs. 1(a)-(d)], the RCP and LCP components are coupled only via the XPM term, which becomes significant only close to the collapse point. While a low input power excitation [Figs. 1(a,b)] results in beam diffraction, the collapse occurs after a finite propagation distance [for instance, $z=7.3$ in Fig. 1(c)] when the input power exceeds the critical level [8]. A substantial converging portion of the phase fronts emerges near the collapse point, see Fig. 1(d). The collapse can be accelerated by the in-

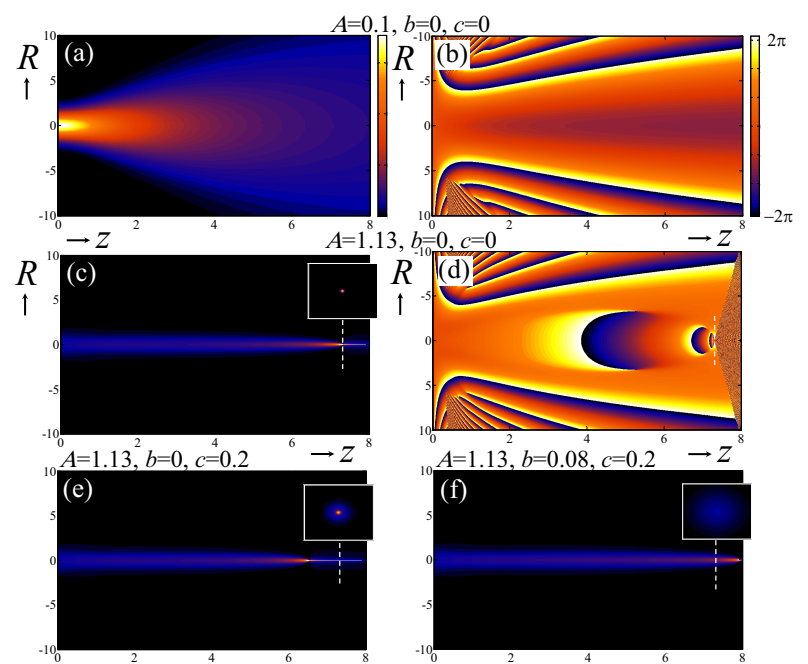

FIG. 1: (Color online) Propagation maps of $u_{r}(R, z)$ obtained from direct solutions of Eq. (1). (a)-(d): Zero birefringences, with (a),(b) low and (c),(d) high input powers. The pairs of panels (a),(c) and (b),(d) display the evolution of the intensity, $\left|u_{r}(R, z)\right|^{2}$, and phase of $u_{r}(R, z)$, respectively. (e), (f): Intensity maps in the presence of the birefringences. Numerical parameters are indicated above each respective panel.
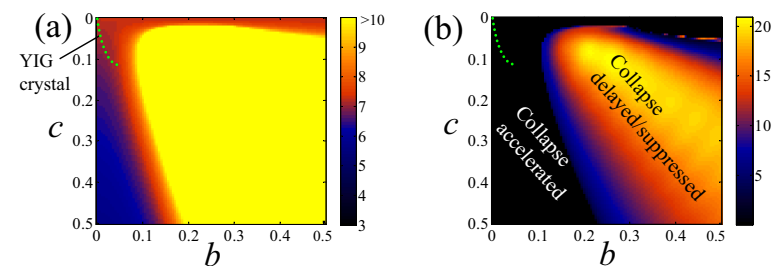

FIG. 2: (Color online) Evolution maps in the parameter space $(b, c)$ (see the text), as obtained from direct simulations of Eqs. (1), for $A=1.13$ and $0 \leq z \leq 50$, up to $z=50$ or the value $z_{\text {coll }}<50$ in which the collapse occurs. The solutions that do not collapse up to $z=50$ are represented in yellow areas. The dashed (green) curves show the calculated dependence between $b$ and $c$ corresponding to crystalline YIG in an external magnetic field. (a) $z_{\text {coll }}$, and (b) beam width at $z_{\text {coll }}$.

troduction of linear birefringence, as shown in Fig. 1(e). Circular birefringence, if acting alone, does not affect the collapse, since the respective terms in Eqs. (1) can be eliminated by a straightforward transformation. When both of the birefringences are present, the propagation distance necessary for the onset of collapse can be $e x$ tended, i.e., the onset of the collapse is delayed [Fig. 1(f)] due to the interplay between amplitude and phase mixing. For low $b$ and $c$ values, such as those used in Fig. $1(\mathrm{e}, \mathrm{f})$ and typically achievable in experiment, the differences in the evolution of $u_{r}$ and $u_{l}$ are marginal, i.e., the RCP and LCP beam components feature the same collapse dynamics. With larger birefringence parameters the differences between the components become substantial; however, such large birefringence values were not accessible in the current experiment, see below.

The results of systematic simulations are summarized in Fig. 2 by means of maps in the plane of $(b, c)$. Panels (a) and (b) show, respectively, the values of $z_{\text {coll }}$ where the beam collapses, if $z_{\text {coll }}<50$, and the final values of the beam width, $\rho(z=50)$; in cases where the collapse occurs at $z_{\text {coll }}<50$, the final width is set as $\rho=0$ [black areas in (b)]. While Figs. 1 and 2 display the results for the RCP component, the LCP maps are similar in the entire range considered. As seen in Fig. 2, for a given input power the domination of amplitude mixing between RCP and LCP $(c>b)$ usually results in an acceleration of the collapse, while dominant phase mixing $(b>c)$ can lead to the delay or effective suppression of the collapse.

In the experiment, a bulk YIG single-crystal was placed with the easy crystallographic axis [100] parallel to $z$, cf. Ref. [17]. These cubic dielectric crystals are highly transparent for optical signals in the near-infrared and exhibit large MO transmission coefficients, owing to their ferrimagnetic phase [15, 16]. We chose to work at the wavelength of $1.2 \mu \mathrm{m}$, which offers an optimal tradeoff between the magnitudes of the MO coefficients and the absorption losses 16. The temporal dispersion in YIG is normal and weak in the near-infrared [18], and hence a high power beam is free from the development of 


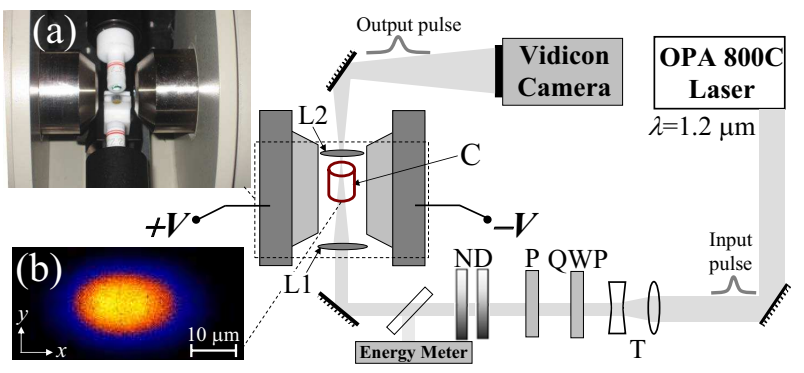

FIG. 3: (Color online) Experimental setup schematics. OPA, optical parametric amplifier laser; $\mathrm{T}$, cylindrical telescope; QWP, quarter wave plate; $\mathrm{P}$, polarizer; ND, neutral density attenuators; L1,L1, aspheric coupling and imaging lenses; C, crystal sample. Insets: (a) Photograph of the magnet's interior with lenses L1, L2 and crystal C; (b) The beam's waist profile at the input facet of the crystal.

temporal modulational instabilitiess [7].

The application of an external magnetic field $\mathbf{H}$ perpendicular to the propagation axis renders the crystal optically uniaxial, with the optical axis parallel to $\mathbf{H}$ and the respective linear birefringence proportional to $H$ [14, 15]. The largest phase retardation associated with the linear birefringence, for components of the wavevector $\mathbf{k}$ perpendicular to the magnetic field, is $0.45 \mu \mathrm{m} / \mathrm{cm}$ in YIG [15] at a saturation field of $H=800 \mathrm{G}$. This corresponds to a saturation value of $c=0.1$ in terms of Eq. (1) for our crystal. For wavevector components parallel to the magnetic field, the Faraday effect induces different refractive indices for the RCP and LCP waves (i.e., circular birefringence), with a respective polarization phase retardation of $2 \mu \mathrm{m} / \mathrm{cm}$ at the saturation level [16]. While the Faraday effect is predominant in the $\mathbf{k} \| \mathbf{H}$ geometry [17], it is weaker in the present setting, as only near the collapse point the wavevectors feature significant lateral components. An estimation corresponding to the case shown in Figs. 1(c),(d) yields an effective saturation value of $b=0.05$. Below saturation, the Faraday coefficient is proportional to the magnetic field, $b \sim H$, while the Cotton-Mouton coefficient depends on $H$ quadratically, i.e. $c \sim H^{2}[14,[15$, 16], implying a parabolic dependence between the birefringence coefficients in a given external magnetic field, $b \sim c^{2}$. Calculated relations between $b$ and $c$ for the YIG crystal at different magnetic fields are shown by the dashed curves in Fig. 2 .

The experimental setup is sketched in Fig. 3. We used a Spectra Physics model 800C Optical Parametric Amplifier laser system, delivering pulses of 200 fs duration at a repetition rate of $1 \mathrm{KHz}$ with peak powers $\leq 50$ MW. The input beam was shaped by means of a cylindrical telescope $(\mathrm{T})$, followed by the combination of a quarter wave plate $(\mathrm{QWP})$ and a polarizer $(\mathrm{P})$, which were used to fix the input linear polarization parallel to $\mathbf{H}$ (i.e. horizontal). Variable neutral density filters (ND) were used to control the input power. The YIG sample

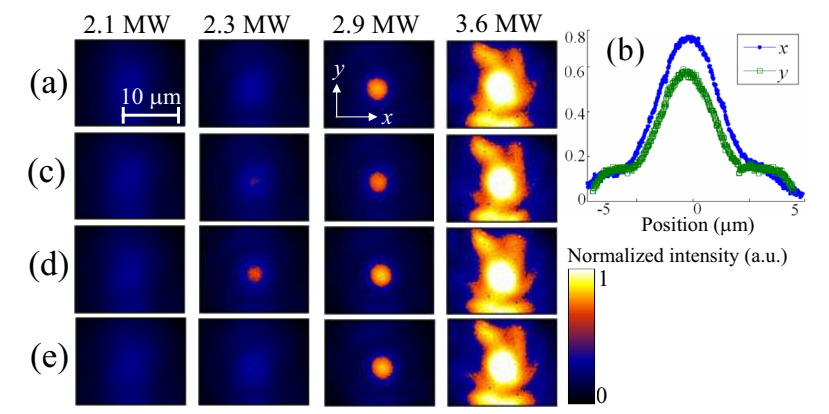

FIG. 4: (Color online) (a) Output beam profiles as a function of the input peak power, in the absence of an external magnetic field. (b) Line-outs along $x$ (full circles) and $y$ (hollow rectangles) around the central spot of the collapsed (2.9 MW) output beam. (c)-(e) Output beam images, as function of the input power (horizontal) and the external magnetic field (vertical), for the fields: $H=200 \mathrm{G}$ (c), $400 \mathrm{G}$ (d), 800 $\mathrm{G}(\mathrm{e})$.

was placed inside an electromagnet (GMW model 3470). The application of a driving voltage between the poles $( \pm V)$ induced a uniform out-of-plane dc magnetic field in the crystal. A digital Tesla-meter (Group3 DTM-133) was used to calibrate the free-space magnetic field and verify its homogeneity in the sample. A combination of aspheric lenses, L1 and L2, was used for coupling and imaging, respectively. Importantly, the lenses and crystal were mounted on nonmagnetic pyrex holders, see Fig. 3(a). Standard metallic mounts, which usually hold microscope objective lenses, were not used, as we found that they strongly affect the uniformity of the magnetic field within the sample. The sample input facet was placed at the focal plane of L1. The beam's profile at this plane is shown in Fig. 3(b). The FWHMs of this elliptical input beam were 30 and $15 \mu \mathrm{m}$ along the major $(x)$ and minor $(y)$ axes. The beam profile at the sample output facet was imaged by L2 onto a Vidicon infrared camera.

Experimental results are summarized in Fig. 4. Images of the output beam as a function of the input peak power, in the absence of a magnetic field, are shown in Fig. 4(a). At the critical power of $2.9 \mathrm{MW}$, a central high-power spot appears in the diffracting background. This spot is circularly symmetric [see Fig. 4(b)], although the input beam profile was elliptical, with the above-mentioned major-to-minor axes ratio of $2: 1$. The spot fits a Townes profile [6], which indicates that it is generated by the onset of the collapse. The corresponding critical power complies with the theoretical prediction for collapse [8], using $\lambda_{0}=1.2 \mu \mathrm{m}$, the linear refractive index of YIG $n_{0}=1.83$ and its Kerr coefficient $n_{2}=7.2 \times 10^{-16} \mathrm{~cm}^{2} / \mathrm{W}[18$. The corresponding collapse distance prediction with $A=1.13, z_{\text {coll }}=7.3$ [Figs. $1(\mathrm{c}),(\mathrm{d})]$, when rescaled back into physical units using the well-known transformations [6, 7, 8] and assuming a circular input beam of $10 \mu \mathrm{m}$ diameter, matches the 

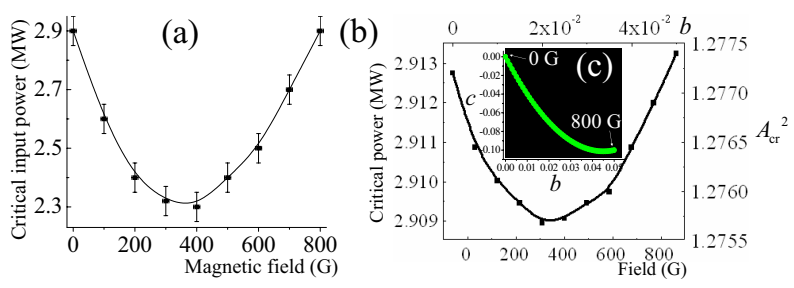

FIG. 5: (Color online) (a) Measured critical power required for the onset of the collapse in the output facet, as a function of the applied magnetic field. (b) Numerically calculated squared critical amplitude of the input beam $\left(A_{\mathrm{cr}}^{2}\right)$ necessary for the onset of the collapse at $z=7.3$, as a function of the birefringences corresponding to crystalline YIG. (c) The calculated dependence between $b$ and $c$ in YIG, used in (b).

sample length, $3 \mathrm{~mm}$. At higher powers, filaments are observed in the output facet images, indicating that the collapse occurred earlier in the crystal. Following the application of a magnetic field, the onset of collapse at the output facet is observed at lower powers [Figs. 4(c-e)], with the largest collapse-acceleration effect recorded at a magnetic field of $H=400 \mathrm{G}$ [see Fig. 4(d)], corresponding to half the saturation field of YIG 17]. This observation agrees with the fact that, when YIG is excited by light traveling perpendicular to the magnetic field, the linear birefringence is stronger than its circular counterpart [14, 15]. A qualitative characterization of the output beam polarization state has also shown that without an external magnetic field the beam remained linearly polarized, while with an application of the field the beam became elliptically polarized, with the ellipticity growing with the field. This indicates the certain presence of magnetically-induced polarization dynamics in the crystal.

Figure 5(a) shows the measured critical power, corresponding to the data of Fig. 4, that was required for the onset of collapse at the output facet, as a function of the magnetic field. In Fig. 5(b), this is compared to results obtained by the numerical solutions of Eqs. (1), where for each set of birefringence values (corresponding to a given magnetic field) we find the critical amplitude $A_{\text {cr }}$ of the input beam for which the collapse occurs at the propagation distance corresponding to the output facet $(z=7.3)$. Figure 5(c) again displays the relation between the normalized birefringence parameters of the YIG crystal used, cf. the dashed curves in Fig. 2. The behavior of the collapse detuning is similar in Figs. 5(a) and 5(b), even though the theoretical model did not take into consideration magnetically-induced losses. Specifically, the Cotton-Mouton effect is always accompanied by linear magnetic dichroism [15], and the Faraday effect entails circular magnetic dichroism [16, 17], both of which are weak but present at $\lambda=1.2 \mu \mathrm{m}$.

In conclusion, we have investigated the combined effects of circular and linear birefringences on the propa- gation of collapsing $(2+1) \mathrm{D}$ beams in self-focusing bulk Kerr media, and have shown that the onset of collapse can be accelerated, delayed, or suppressed, depending on the relative birefringence strengths. Experimentally, we have demonstrated a controlled acceleration of the collapse at the output facet of a ferrimagnetic YIG crystal, following the application of an external magnetic field which induces the birefringences. The direct observation of magnetization-induced effects in collapsing beams provides a unique demonstration of an all-optical magnetically-controlled lensing mechanism, pioneering the use of MO crystals in nonlinear optics experiments. Finally, since Eqs. (11) are also Gross-Pitaevskii equations describing a binary BEC with linear interconversion [19], accounted for by the coefficient $c$, similar phenomena may also be observed in nonlinear matter-wave dynamics.

This research was supported by NSERC and TeraXion (Canada). YL and KR respectively acknowledge support from FQRNT-MELS and IOF Marie Curie fellowships.

* Corresponding author: yoli@emt.inrs.ca.

[1] P. A. Robinson, Rev. Mod. Phys. 69, 507 (1997).

[2] L. Bergé, Phys. Rep. 303, 260 (1998).

[3] B. W. Zeff, B. Kleber, J. Fineberg, and D. P. Lathrop, Nature (London) 403, 401 (2000).

[4] E. A. Donley et al., Nature 412, 295 (2001); M. Greiner, O. Mandel, T. W. Hansch, and I. Bloch, Nature 419, 51 (2002); T. Lahaye et al., Phys. Rev. Lett. 101, 080401 (2008).

[5] J. L. Roberts et al., Phys. Rev. Lett. 86, 4211 (2001); T. Koch et al., Nature Phys. 4, 218 (2008).

[6] K. D. Moll, A. L. Gaeta, and G. Fibich, Phys. Rev. Lett. 90, 203902 (2003).

[7] L. T. Vuong et al., Phys. Rev. Lett. 96, 133901 (2006); T. D. Grow, A. A. Ishaaya, L. T. Vuong, and A. L. Gaeta, ibid. 99, 133902 (2007); L. Bergé et al., Rep. Progr. Phys. 70, 1633 (2007).

[8] G. Fibich and A. L. Gaeta, Opt. Lett. 25, 335 (2000).

[9] G. Fibich et al., Opt. Express 14, 4946 (2006); S. Eisenmann et al., Opt. Express 15, 2779 (2007).

[10] I. Towers and B. A. Malomed, J. Opt. Soc. Am. B 19, 537 (2002).

[11] H. Saito and M. Ueda, Phys. Rev. Lett. 90, 040403 (2003); F. K. Abdullaev, J. G. Caputo, R. A. Kraenkel, and B. A. Malomed, Phys. Rev. A 67, 013605 (2003).

[12] R. W. Boyd, Nonlinear Optics (Academic Press, San Diego, 2008), 3rd ed., Chaps. 1-4.

[13] S. Trillo, S. Wabnitz, E. M. Wright, and G. I. Stegeman, Opt. Commun. 70, 166 (1989); Y. Bar-Ad and Y. Silberberg, Phys. Rev. Lett. 78, 3290 (1997).

[14] R. Kurzynowski and W. A. Wozniak, Optik 115, 473 (2004).

[15] J. F. Dillon, J. P. Remeika, and C. R. Staton, J. Appl. Phys. 41, 4613 (1970).

[16] G. B. Scott, D. E. Lacklison, H. I. Ralph, and J. L. Page, Phys. Rev. B 12, 2562 (1975).

[17] Y. Linzon et al., Opt. Lett. 33, 2871 (2008).

[18] M. J. Weber, Handbook of optical materials (CRC Press, 
2002).

[19] R. J. Ballagh, K. Burnett, and T. F. Scott, Phys. Rev.

Lett. 78, 1607 (1997). 